\title{
Imaging of Electrons with Tracking and Counting Detectors for (S)TEM Applications
}

Björn Eckert ${ }^{1}$, Lothar Strueder ${ }^{2}$, Stefan Aschauer ${ }^{1}$, Petra Majewski ${ }^{1}$ and Thomas Zabel $^{1}$

${ }^{1}$ PNSensor, Munich, Bayern, Germany, ${ }^{2}$ PNSensor and University of Siegen, Munich, Bayern, Germany

Event detection with a good spatial resolution, a high contrast, and low noise is required for direct electron detection in many transmission electron microscopy applications. A precise reconstruction of the point of entry $(\mathrm{PoE})$ of the primary electron into a pixelated detector is crucial. However, higher energetic electrons penetrate matter and do not deposit their energy completely at their PoE, but produce tracks in the detector volume often extending over many pixels. The length of these tracks strongly depends on the primary energy of the electrons. Statistically, an electron deposits most of the energy at the end of its track. As a consequence, the PoE and the maximum energy deposition is spatially separated.

A distinction between two readout concepts for pixelated detectors can be made: With tracking detectors, the tracks of the electrons with their corresponding energy depositions are extracted, whereas counting detectors only store the position of those pixels where an energy deposition exceeds a certain threshold. Moreover, two parameters are decisive for the correct event detection. On the one hand, the local accuracy of the reconstructed $\mathrm{PoE}$ and on the other hand the ratio between the number of detected events and real events. These two quantities depend on the type of detection and reconstruction method, but also on properties such as the pixel size, the electron rate or the primary energy. To compare these parameters over a wide band of primary electron energies Monte Carlo simulations (GEANT4) were performed with a detector pixel size of $48 \times 48 \mu \mathrm{m}^{2}$ to extract key figures of merit.

For counting, the multiplicity in Fig. 1.a indicates how many pixels are hit by one primary electron on average. For thresholds, of the order of the total detection noise, the municipality diverges. In this case, also pixels are counted as hits, which have only detected noise but no energy deposition of the primary electron. For thresholds, which are much larger than the noise, the multiplicity is larger for higher energies of the primary electron than for small primary energies, since the energy is deposited in a larger volume. Especially for high energies of the primary electron, the multiplicity decreases with higher thresholds. Here the high threshold acts as an event filter. Primary electrons, which distribute their energy over a larger volume, i.e. more pixels, are not detected by this filter with a higher probability.

The detective quantum efficiency (DQE) is a measure of the detector system influence on the signal to noise ratio. A DQE of one means that the signal to noise ratio of the number of primary electrons (Poisson distributed) is not changed by the detector system. Assuming that the total system noise is small compared to the deposited energy for each pixel, and assuming that the tracks of each primary electron are separable, the DQE is one for tracking systems. In this case, each primary electron is counted exactly once. The DQE of counting systems strongly depends on the selected threshold (Fig. 1.b). For low energy thresholds, the DQE tends to be higher for all primary energies. The DQE for low primary energies has a plateau. At higher thresholds, fewer pixels can contain energies higher than the threshold. This leads to a lower signal to noise ratio at the output of the detector system and a decreasing DQE. The plateau is not so pronounced for higher primary energies since the variation of counted pixels per primary electron is higher. This leads to higher noise and respectively to a lower signal to noise ratio at the output.

To measure the spatial resolution the Euclidean distance between the true PoE and the predicted PoE was calculated. For counting, the distances of all pixels above the selected threshold were treated separately. The distance to the true PoE for counting is presented in Fig. 2.a. For higher primary energies, first, the distance to the true PoE increases with higher energy thresholds. This phenomenon can be explained by looking at the deposited energy distribution along the track. At the beginning of the track, only a small 
amount of energy is deposited. On average, pixels that are near the PoE have a small amount of deposited energy. With an increase of the threshold, these pixels are no longer considered. Only pixels far away from the PoE remain. Upon further increasing the threshold, it additionally functions as an event filter. This event filter only permits events in which the energy deposition concentrated in few pixels. For lower primary energies, a similar event filter leads for higher thresholds to a decreasing distance to the true PoE. For tracking systems, various methods for the reconstruction of the PoE were used (Fig. 2.b). For low primary energies, the weighted centroid method performs best, whereas for higher energies the "furthest pixel away" method [1] gives the best results.

In summary, an application-related choice of threshold is decisive. At high primary energies, better resolutions can be achieved with tracking systems than with pure counting systems.

a

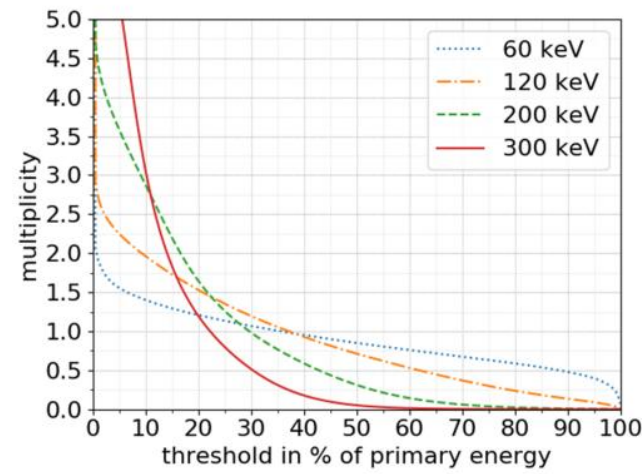

b

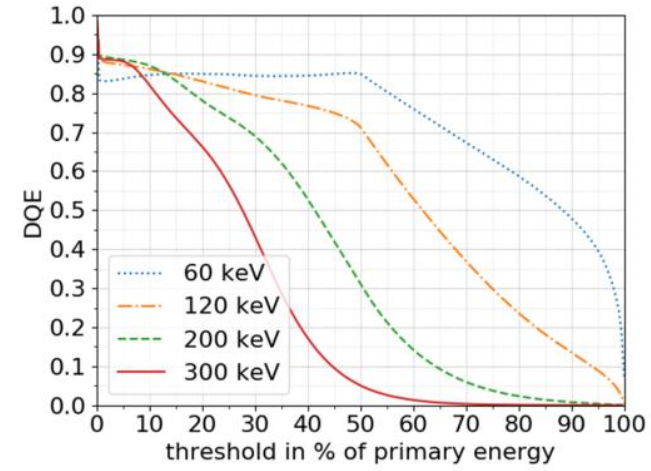

Figure 1. a: The multiplicity for various energies of the primary electron as a function of the threshold. Fig. 1.b: The DQE for various energies of the primary electron as a function of the threshold for counting detector systems.

a

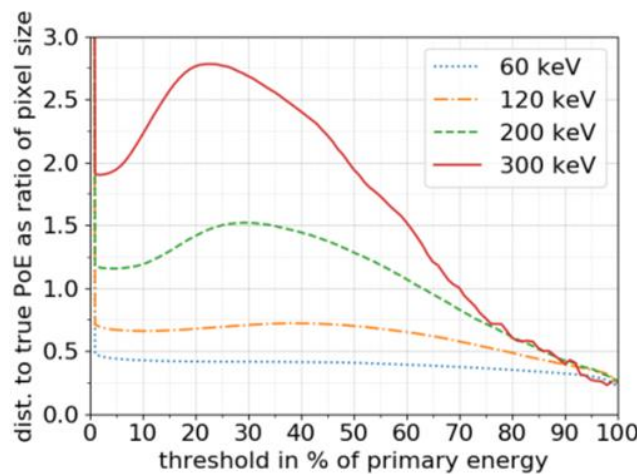

$\mathrm{b}$

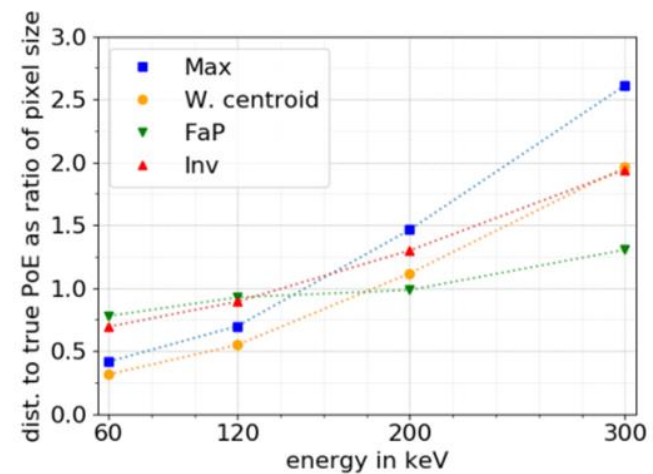

Figure 2. a: The averaged Euclidean distance of the predicted PoE to the true PoE for various primary electron energies as a function of the pixel by pixel energy threshold. Fig. 2.b: The averaged Euclidean distance of the predicted PoE to the true PoE for different reconstruction methods of tracking data. Max: The pixel with the maximal energy deposition is used as predicted PoE. W. centroid: The energy weighted centroid is used as predicted PoE. FaP: The pixel furthest away from the pixel with the maximal energy deposition of the event is used as predicted PoE. Inv: The inverse energy weighted centroid is used as predicted PoE.

\section{References}

[1] H. Ryll et al., A pnCCD-based, fast direct single electron imaging camera for TEM and STEM, Jinst., Volume 11, Issue 04, pp. P04006 (2016) 